\title{
Crystal Methamphetamine Use and HIV Transmission Among Gay and Bisexual Men
}

\author{
Perry N. Halkitis • Michael D. Levy • Alvaro D. Moreira • Charles N. Ferrusi
}

Published online: 3 June 2014

(C) Springer International Publishing AG 2014

\begin{abstract}
In the United States, methamphetamine continues to be a substance that is used and abused by gay, bisexual, and other men who have sex with men (MSM). Although there is no evidence that suggests a causal pathway between use of the drug and HIV seroconversion, the two are highly associated. Moreover, numerous studies indicate use and abuse of methamphetamine among gay and bisexual men even after seroconversion. Effective interventions for treating the addiction must fully address the 'meth-sex' link. While strides have been made during the last decade in addressing methamphetamine addiction, the problem perpetuates within the population. It is proposed that future efforts must consider methamphetamine addiction more holistically within the larger framework of the totality of health disparities faced by sexual minority men, which includes, but is not limited to, HIV, and which understands and seeks to address the social and psychosocial stressors experienced by these men, which makes them vulnerable to the addiction.
\end{abstract}

Keywords Men who have sex with men · MSM · HIV seroconversion · Addiction · Methamphetamine abuse · Sexual minority men

\section{Introduction}

During the course of the last two decades, the illicit drug methamphetamine (i.e. crystal meth, Tina, crank, ice) has

\section{P. N. Halkitis $(\square)$}

Center for Health, Identity Behavior \& Prevention Studies, Global

Institute of Public Health, and Department of Population Health,

Langone Medical Center, New York University, 726 Broadway,

Suite 525, New York, NY 10003, USA

e-mail:pnh1@nyu.edu

M. D. Levy • A. D. Moreira • C. N. Ferrusi

Center for Health, Identity Behavior \& Prevention Studies,

New York University, New York, NY, USA garnered much attention in the behavioral and public health literature, particularly as it relates to use, abuse, and addiction in the population of gay and bisexual men. Despite historical evidence which suggests that the drug has been used and abused in the United States (US) for more than 50 years, the prevalence of use among sexual minority men has been an area of concern because of the close association between use of the drug and the transmission of sexual pathogens, particularly HIV $[1,2]$. It has been proposed that the hypersexualinducing effects of the substance [3] which may be exacerbated by urban gay culture $[4,5]$ increase the likelihood of HIV transmission, in effect creating a 'dual epidemic' [6].

Data from the National Survey on Drug Use and Health [7] indicate that 1.2 million people ( $0.4 \%$ of the population) in the US reported using methamphetamine in 2012. Moreover, 133,000 individuals aged 12 years and older initiated use of methamphetamine that year, identical to the estimate of initiation in $2011(133,000)$ and slightly more than the rate in 2010 $(107,000)$. Age of initiation was estimated at 19.7 years, which is consistent with age of initiation over the course of the preceding decade. Rates of use of methamphetamine are comparable across genders [8].

The use of methamphetamine in the US population is also indicated by the Treatment Episodes Data Set (TEDS) [9]. According to the TEDS, in 2007 approximately 143,024 individuals were admitted for the treatment of amphetamine, including methamphetamine, as the primary substance of admission, with the majority of admissions being among those under 40 years of age, and with smoking noted as the primary route of administration in most of these admissions (66.4\%). Moreover, $11.1 \%$ of females were admitted with methamphetamines/amphetamines as their primary substance at admission as compared to $6.3 \%$ of all male admissions. Admissions data also indicate the prevalence of methamphetamine use across race/ethnicity among White non-Hispanics. Overall, $8.7 \%$ of all admissions were for addiction to 
methamphetamine/amphetamine admissions; $1.1 \%$ for Black non-Hispanics, $11.6 \%$ for Hispanics, and 14.8\% for others, including Asian Pacific Islanders, Alaska Natives, and American Indians (14.8 \%). Between 2001 and 2001, admissions for methamphetamine/amphetamine for those aged 12 years and over rose from 40 per 100,000 to 42 per 100,000 , peaking in 2005 at 69 per 100,000 [10].

Despite this abundance of epidemiological data on the use and abuse of methamphetamine in the US, no such comprehensive information is available for the population of gay and bisexual men. Until recently, measures of sexual orientation were not included in large-scale surveys, although recently there has been an increase in the inclusion of such measures in state surveys in the US [11]. Still, to date, most data available on the pervasiveness of methamphetamine use in the gay and bisexual male population in the US are derived from smallerscale behavioral studies [2], and there continues to be a need for population-based data on the use of this and other substances [12]. Despite these lack of data, Reback et al. [13] suggest that gay, bisexual, and other men who have sex with men (MSM) report rates of use to 20 times greater than that of the general population.

In what follows, we provide an overview of recent findings, which describe how methamphetamine use manifests in the lives of gay and bisexual men. We draw upon recently published empirical studies to (1) depict patterns of methamphetamine use; (2) examine the link between methamphetamine use and sexual risk-taking behavior; and (3) consider demonstrated and effective treatment modalities for addressing the methamphetamine-sex link in the population.

\section{Use of Methamphetamine in Gay and Bisexual Men}

In a study of methamphetamine use and cognitive function, Solomon et al. [14] indicated relatively high rates of use of the drug among gay and bisexual men. Of 215 men screened for eligibility in this study, $99.5 \%$ of whom identified as gay or bisexual and who were, on average, 39 years of age, $74 \%$ indicated lifetime use of methamphetamine. Lifetime use of the drug was highly associated with the use of other substances, including ecstasy, ketamine, and crack cocaine. Differences emerged across race ethnicity, with $81.8 \%$ of Black men, $62.5 \%$ of White men, and $83.7 \%$ of Hispanic men reporting lifetime use; this was higher among HIVpositive men than HIV-negative men. With regard to HIV status, Halkitis et al. [15•] also recently reported that methamphetamine use does not always precede seroconversion; among the 58 men in a sample of active methamphetamine users who reported being HIV-positive, $65 \%$ reported onset of methamphetamine use following seroconversion.

Use of methamphetamine transgresses age in the population of gay and bisexual men. A study of adolescent and young MSM (YMSM) provides an indication of methamphetamine in this younger segment of the population [16•]. In a sample of 595 YMSM drawn across eight cities, 64 (11\%) indicated use of methamphetamine in the 90 days prior to assessment, and among those who had indicated use of drug ( $n=187), 35.4 \%$ reported using methamphetamine. Of these 64 YMSM, $15.6 \%$ reported no other drug used in the time period of assessment, while $28.1 \%$ reported the injection of illicit drugs. The methamphetamine-using YMSM tended to be older than non-drug users in the sample, were less likely to be attending school, and were more likely to have been homeless and have had a history of a sexually transmitted infection (STI). All but one of the methamphetamine users was aged 18 years or older, and users of the drug represent diversity in terms of race/ethnicity: White $(33.4 \%)$, Black (12.5\%), Hispanic $(39.1 \%)$, other/mixed race $(14.1 \%)$. In a similar sample of 540 YMSM aged 18-29 years, in New York City [17•], recent use (defined as 3 months prior to assessment) was reported by approximately $3 \%$ of the sample. However, use varied by context of social navigation, with $8.5 \%$ of those who reported attending sex parties also reporting methamphetamine use as compared with only $2 \%$ who did not report sex party attendance.

The role of social context, and in particular social networking, has been examined in relation to methamphetamine use among MSM. Young and Shoptaw [18] reported on a sample of 118 African American and Latino MSM, the majority of whom utilize social networking technologies to meet people, and $30 \%$ of whom use these sites for identifying sexual partners. Approximately $15 \%$ of the sample reported use of methamphetamine in the year prior to assessment and $8.9 \%$ in the prior month. Findings further indicate that those who used social networking to meet sexual partners had a higher likelihood of using methamphetamine. Moreover, those who indicated greater comfort using online systems to meet people (as compared to in person) reported a four times greater odds of methamphetamine use in the year prior to assessment.

The role of the Internet in relation to methamphetamine use was also examined in a sample of HIVinfected individuals seeking medical care in San Francisco [19•]. During 2008, 435 gay men were recruited at two healthcare sites. Of these men, $35 \%$ reported use of methamphetamine in the prior 12 months and $23 \%$ in the prior 4 weeks. Moreover, $55 \%$ reported both methamphetamine use and Internet use for identifying sex partner in the past year, while $27 \%$ reported this in the last 4 weeks. In another study of HIV-positive methamphetamine-using MSM, Nakamura et al. [20•] reported an average of 10.43 days of drug use among the 297 participants. Similarly, in a study of Black methamphetamine-using MSM, Jerome et al. [21•] reported an average of 8.61 days of use (Median $(\mathrm{Md})=\$ 6)$, with a monthly financial liability of $\$ 150(\mathrm{Md}=\$ 65)$. 


\section{Methamphetamine Use and Sexual Risk-Taking in Gay and Bisexual Men}

Recent literature has confirmed what has previously been documented about methamphetamine in the context of sexual risk behavior and HIV seroconversion. Studies have demonstrated that among gay, bisexual, and other MSM, use of methamphetamine is associated with a constellation of sexual risk behaviors.

The link between methamphetamine use and unprotected sex in gay, bisexual, and other MSM is a complex phenomenon. Facilitators of methamphetamine use among MSM have been noted as both psychological (e.g. decreased inhibition, increased libido), physiological (e.g. decreased fatigue, increased sensory), and situational (e.g. venues, social environment) [22•]. Methamphetamine use and unprotected sex are fueled by the effects of the drug, which include prolonging sexual performance, increasing sexual enhancement, and heightening sexual desire. These conditions can all lead to higher levels of sexual risk behaviors, placing HIV-negative MSM at higher risk for contracting HIV, and HIV-positive MSM at higher risk of transmitting it. The use of this drug comes with various subsequent effects that moderate and modify the link between methamphetamine, unprotected sex, and HIV transmission $[16 \bullet]$.

\section{Methamphetamine Use and Unprotected Sex}

The association between methamphetamine use and unprotected anal intercourse is well documented among MSM. A systematic review examining substance use and sexual risk behavior among MSM measured 15 substances and the associated sexual risks [22•]. Methamphetamine use was one of two substances (the other being binge drinking) that showed consistent association with sexual risk behavior. Methamphetamine use preceding sex was evaluated in 8 of the 35 studies utilized in this review, and these findings indicated an association with receptive unprotected anal intercourse, a behavior considered high-risk for HIV transmission. Moreover, even when controlling for other factors, the link between sexual risk behavior and methamphetamine use remained consistent.

Such findings are corroborated among HIV-positive MSM, where a strong association has been detected between the use of methamphetamine during sex with both multiple sex partners and the likelihood of engaging in unprotected anal intercourse [23•]. In another study, one-third of HIV-positive MSM reported methamphetamine use and unprotected anal intercourse, a pattern that remained consistent over time [24]. In a sample of racially diverse HIV-positive MSM in medical care in San Francisco, methamphetamine users reported an increased rate of unprotected sex among [19॰]. Specifically, of those who have used methamphetamine in the past 12 months, $27 \%$ reported unprotected sex sometimes, often, or always as compared with $10 \%$ who did not use the drug. Additionally, methamphetamine users reported a higher number of sexual partners.

The link between methamphetamine use and unprotected sex is also noted among young men. Among 12- to 24-yearold YMSM across eight cities, methamphetamine users were observed to have higher rates of unprotected sex, higher likelihood of having a history of STIs, and higher number of sex partners in the 90 days prior to assessment as compared with those who did not use methamphetamine [16•]. Participants who used methamphetamine in the past 90 days were also identified as older and more likely to be homeless. Freeman et al. [16•] also found that users of methamphetamine increased their risk by engaging in sex with HIVpositive partners and intravenous drug users at higher rates than their non-using counterparts. Moreover, two-thirds of methamphetamine users did not consistently use condoms. Notably, and possibly due to age effects and despite an elevated level of sexual risk behaviors, only $10 \%(n=40)$ of the YMSM were HIV-positive. These elevated risk behaviors, although co-occurring and implicitly related, make it difficult to disentangle how these behaviors interact, especially chronologically, to create new infections of HIV among gay, bisexual, and other MSM. Establishing a direct causal link between methamphetamine use and consequential HIV transmission has proven a more difficult task to evidence and clarify.

\section{Methamphetamine and HIV Transmission}

While research documents a strong association between methamphetamine use and sexual risk behaviors in gay, bisexual, and other MSM of all ages, it is difficult to disentangle how exactly these behaviors interact, especially chronologically, to create new infections of HIV in the population. Thus, establishing a direct causal link between methamphetamine use and proximal HIV transmission has proven a difficult task to evidence and clarify.

Only one study in recent years has explicitly addressed the temporal relationship between methamphetamine initiation and HIV seroconversion in gay, bisexual, and other MSM. While prior research has implied a linear progression from methamphetamine use to HIV serconverison, Halkitis et al. [15•] found that $65 \%$ of HIV-positive methamphetamine users began using the substance only after they had seroconverted, and 8 years separated these two events for the men in this sample. Moreover, among those who seroconverted after methamphetamine imitation, there was a 9-year gap between onset of use and HIV seroconversion. Such results suggest that use of methamphetamine does not always precede seroconversion and, moreover, use of the drug 
does not always immediately lead to the acquisition of HIV, despite the manner in which this relationship is popularly depicted.

Interventions Addressing the Methamphetamine-Sex Link in Gay and Bisexual Men

During the last several years, interventions tested on methamphetamine-using gay, bisexual, and other MSM have sought to reduce HIV transmission by focusing on sexual risk behavior and/or methamphetamine use, using cognitive behavioral therapy (CBT), motivational interviews (MI), contingency management (CM), and/or pharmacological interventions.

Shoptaw et al. and Reback et al. developed and tested a tailored, gay-specific CBT (GCBT) [25, 26]. In a replication study, researchers found that in broader groups of substanceusing gay and bisexual men, both GCBT and the comparator gay social support therapy (GSST) performed equally in reducing substance use during treatment, with GCBT outperforming GSST in reducing methamphetamine use among methamphetamine-abusing gay and bisexual men after 1 year [27]. Reback and Shoptow continued to develop the intervention, reducing it to 8 weeks and 24 sessions, and evaluated the impact of this modification in the size of outcomes in retention, methamphetamine use, and sexual risk behaviors [28]. They also coupled the GCBT with a $\mathrm{CM}$ intervention specifically designed to be cost and time effective for community application. Their findings indicate that while the modified GCBT + CM intervention has slightly less potency at reducing the use of methamphetamine than its progenitor versions, it has consistently stronger effects in reducing the number of male sexual partners, suggesting that most of the benefits of the evidence-based interventions are retained when utilizing the condensed, modified GCBT + CM intervention in a community setting.

Researchers have implemented this modified version without the CM, and renamed it Getting Off: A Behavioral Treatment Intervention for Gay and Bisexual Male Methamphetamine Users [29]. Reducing the size of the program improved the feasibility of a community site to deliver the intervention, and staffing costs were greatly reduced by developing two manuals for the intervention, making it possible to use staff with substance abuse counseling experience to deliver the intervention. Getting Off has been adopted by multiple community-based sites in Los Angeles, where it has been publicly funded since 2007 , and nationally in multiple settings where HIV and methamphetamine use prevalence is high.

Garfein et al. [30•] also focused on behavioral interventions and combined it with MI to evaluate the feasibility and acceptability of an intervention for decreasing unprotected anal intercourse, and increasing awareness of parenteral HIV transmission risk among methamphetamine-using gay, bisexual, and other MSM. Results suggests that, although the proposed intervention topics were considered useful in reducing their sexual risk behaviors, the neurocognitive effects of methamphetamine were reported to be a barrier for safe sex, condom use negotiation, or HIV status disclosure, concluding that the intervention should emphasize pre-planning to avoid combining methamphetamine use and sex or develop strategies to avoid sex risk following methamphetamine use.

A study by Zule et al. [31•] tested a single-session MI intervention for reducing HIV risk among an out-oftreatment sample of methamphetamine-using gay, bisexual, and other MSM, designed to increase self-efficacy of participants to avoid high-risk behaviors. Participants self-reported significant reductions in sexual risk and methamphetamine use between the baseline interview and the follow-up interview 2 months later. They also self-reported a significant increase in the stage of change regarding stopping methamphetamine use and eliminating sexual risk behaviors. These results suggest that this approach may be useful for reducing methamphetamine use and sexual risk among methamphetamine-using gay, bisexual, and other MSM, especially in settings where multi-session interventions are not feasible.

In an exploratory investigation of treatment strategies for Black MSM, Jerome and Halkitis [32] asked participants to discuss methamphetamine treatment strategies they believed would be successful in treating their own methamphetamine abuse. Their findings underscore the importance of adapting evidence-based methamphetamine treatment strategies to include culturally relevant factors that address the specific needs of Black methamphetamine-using gay, bisexual, and other MSM. Along these lines, $\mathrm{Wu}$ et al. [33] conducted a pilot study for a seven-session, couple-based, sexual risk reductions intervention specifically for Black methamphetamine-using same-sex couples, engaging in sexual risk. The structured, manualized intervention used an existing evidence-based intervention, originally developed for heterosexual couples [34], and was adapted to incorporate the world views and lived experiences of Black same-sex couples. At follow-up, participants self-reported significantly fewer sexual partners, fewer episodes of unprotected anal sex, and greater condom use with their main partner; participants also self-reported significantly less methamphetamine use, any illicit drug use, and number of illicit drugs used. These findings indicate that couple-based HIV/STI intervention is feasible and promising for at-risk Black MSM couples. Such a tailored approach to intervention is focal in the research by Reback et al. [35•] with homeless MSM, who are a particularly vulnerable population with high 
rates of substance dependence, psychiatric disorders, and HIV prevalence.

Considering how methamphetamine-using gay, bisexual, and other MSM use information technology (IT) communication (e.g. instant messaging, social networking sites, websites) to facilitate their sexual and/or drug 'hook ups', an IT intervention using text messaging is an innovative and feasible way to reach and intervene with out-of-treatment methamphetamineusing gay, bisexual, and other MSM. Reback et al. [36•] conducted formative work to develop a 2-week text-messaging intervention that delivered real-time electronic correspondence to reduce methamphetamine use and high-risk sexual behaviors among methamphetamine-using gay, bisexual, and other MSM. Using focus groups, community meetings, and a pre-test, researchers were able to identify the best IT communication device, text messages, as those that best support risk reduction and healthier behavioral choices. They also involved the target population and community partners, whose feedback was used to develop a culturally appropriate intervention. Researchers face the 'time' challenge posed by protocol approval and recruitment versus the rate of technological advancement. When the study was designed, the messaging device considered for use was a two-way pager; however, by the time of its implementation, this device was considered grossly obsolete and research budgets did not allow them to keep up with the speed of technology.

Regarding pharmacological treatments, Das et al. [37] explored the feasibility and acceptability of a pharmacologic intervention trial for methamphetamine dependence in MSM. Findings suggest that it is feasible to enroll and retain actively using, methamphetamine-dependent MSM in a pharmacologic intervention, and that bupropion was well tolerated, although adherence was only moderate.

\section{Conclusion}

Despite the development of treatment approaches that demonstrate some effectiveness, methamphetamine continues to burden the lives of gay and bisexual men. It should be noted that many of these treatments address the proximal behaviors of methamphetamine use and unprotected sex. Yet, one is left to wonder why this drug continues to wreak havoc in the population despite our efforts to address the addiction, and the community action that has evolved over the course of the last decade [38].

Perhaps our ability to address the 'meth-sex' link and to alleviate the population of this burden, our work must be rooted in fully understanding why gay and bisexual men are drawn to methamphetamine, specifically, and substance use more broadly [1]. It is posited that the initiation of methamphetamine may help some gay and bisexual men confront issues of self-esteem, guilt, and shame associated with sexual identity, and may serve as a means of coping with the stigma, internalized homophobia, lack of sense of belonging, and minority stress experienced by some [39•, 40, 41•, 42, 43]. For many gay and bisexual men, these socially constructed burdens associated with their sexual orientation are exacerbated by poverty [44], racial and ethnic discrimination [15•], and homelessness [13].

Taken together, such psychosocial factors may explain the elevated levels of depression noted among gay and bisexual men who evolve into methamphetamine users [45•]. For HIVpositive men, methamphetamine may also serve as a mechanism to confronting their disease [15•], especially for those who are long-term survivors and whose elevated levels of trauma are evident from having lived through the height of the AIDS epidemic [46]. Social conditions may also explain the high rates of methamphetamine use reported in the aforementioned studies among the sexual minority men of color, who experience multiple forms of oppression and discrimination, including, but not limited to, their sexual orientation, race/ethnicity, and economic status [41•]. Thus, in the absence of effective social and mental health support and in light of the discrimination faced by many gay and bisexual men [40], methamphetamine may serve as a tool, albeit a limited an ultimately ineffective one, for addressing the ongoing mental health burdens experienced by gay and bisexual men.

Our ability to confront the health challenges faced by gay and bisexual men must be undertaken using holistic approaches that recognize that HIV disease and methamphetamine addiction do not exist in isolation, and that these health challenges interact themselves and with other health conditions, including mental health burden and violence, experienced by gay and bisexual men [39॰]. Moreover, these overlapping epidemics are not indicated solely by behaviors of individuals but also the psychosocial and social conditions in which gay and bisexual men live [39•, 47]. Similarly, it is crucially important to address physical and biological aspects of methamphetamine addiction, specifically neurological effects, which have been shown to interfere with interventions aimed at addressing behavior [30•]. In effect, eradicating methamphetamine addiction requires that we adopt a biopsychosocial perspective [39•, 48] which attends equally to physical, emotional, and social well-being, with attention to the social inequities faced by gay and bisexual men [49].

\section{Compliance with Ethics Guidelines}

Conflict of Interest Alvaro Moreira, Charles Ferrusi, Michael Levy and Perry N. Halkitis declare that they have no conflicts of interest.

Human and Animal Rights and Informed Consent This article does not contain any studies with human or animal subjects performed by any of the authors. 


\section{References}

Papers of particular interest, published recently, have been highlighted as:

- Of importance

1. Halkitis PN, Pollock JA, Pappas MK, Dayton A, Moeller RW, Siconolfi D, et al. Substance use in the MSM population of New York City during the era of HIV/AIDS. Subst Use Misuse. 2011;46(2-3):264-73.

2. Halkitis PN. Methamphetamine addiction: biological foundations, psychological factors, and social consequences. Washington, DC: American Psychological Association; 2009.

3. Palamar JJ, Kiang MV, Storholm ED, Halkitis PN. A qualitative descriptive study of perceived sexual effects of club drug use in gay and bisexual men. Psychol Sex. 2014;5(2):143-60.

4. Aguilar JP, Sen S. The culture of methamphetamine: reframing gay men's methamphetamine use. J Hum Behav Soc Environ. 2013;23(3):370-82.

5. Isaiah Green A, Halkitis PN. Crystal methamphetamine and sexual sociality in an urban gay subculture: an elective affinity. Cult Health Sex. 2006;8(4):317-33.

6. Halkitis PN, Parsons JT, Stirratt MJ. A double epidemic: crystal methamphetamine drug use in relation to HIV transmission. J Homosex. 2001;41(2):17-35.

7. Substance Abuse and Mental Health Services Administration. Results from the 2012 national survey on drug use and health: summary of national findings. Substance Abuse and Mental Health Services Administration; 2013. Available at: http://www. samhsa.gov/data/NSDUH/2012SummNatFindDetTables/Index. aspx. Accessed 7 Apr 2014.

8. Durell TM, Kroutil LA, Crits-Christoph P, Barchha N, Van Brunt DL. Prevalence of nonmedical methamphetamine use in the United States. Subst Abuse Treat Prev Policy. 2008; 3(1):19.

9. Substance Abuse and Mental Health Services Administration. Mental Health Services Administration: Treatment Episode Data Set (TEDS)-Highlights 2007. National Admissions to Substance Abuse Treatment Services. Substance Abuse and Mental Health Services Administration, Office of Applied Studies 2009. Available at: http://www.samhsa.gov/data/TEDS2k7highlights/ TEDSHigh2k7.pdf. Accessed 7 Apr 2014.

10. Substance Abuse and Mental Health Services Administration. Treatment Episode Data Set (TEDS) 2010-2011. Substance Abuse and Mental Health Services Administration, Office of Applied Studies; 2014. Available at http://www.samhsa.gov/data/ DASIS/TEDS2011St Web.pdf. Accessed 7 Apr 2014

11. Evans L, Lawler K, Sass S. Gathering sexual orientation data on statewide behavioral risk factor surveillance surveys: a call to action for states. 2014. Available at: http://fenwayfocus.org/wp-content/ uploads/2014/03/BRFSS-brief-March-20-2014-final.pdf. Accessed 1 Apr 2014.

12. Halkitis PN, Moeller RW, Siconolfi DE, Jerome RC, Rogers M, Schillinger J. Methamphetamine and poly-substance use among gym-attending men who have sex with men in New York City. Ann Behav Med. 2008;35(1):41-8.

13. Reback CJ, Peck JA, Fletcher JB, Nuno M, Dierst-Davies R. Lifetime substance use and HIV sexual risk behaviors predict treatment response to contingency management among homeless, substance-dependent MSM. J Psychoactive Drugs. 2012;44(2): 166-72.

14. Solomon TM, Halkitis PN, Moeller RW, Siconolfi DE, Kiang M, Barton S. Sex parties among young gay, bisexual, and other men who have sex with men in New York City: attendance and behavior. J Urban Health. 2011;88(6):1063-75.

15. Halkitis PN, Levy MD, Solomon TM. Temporal relations between methamphetamine use and HIV seroconversion in gay, bisexual, and other men who have sex with men. J Health Psychol. Epub 27 Feb 2014. This analysis examined the onset of methamphetamine use and HIV seroconversion among MSM and indicated that onset of methamphetamine use does not always precede HIV seroconversion. It was also shown that many years may elapse between onset of methamphetamine use and HIV seroconversion. It is posted that use of the drug by HIV-positive individuals may be a coping mechanism for living with the disease.

16. Freeman P, Walker BC, Harris DR, Garofalo R, Willard N, Ellen JM, et al. Methamphetamine use and risk for HIV among young men who have sex with men in 8 US cities. Arch Pediatr Adolesc Med. 2011;165(8):736-40. An observational analysis of eight US cities found that YMSM, aged 12-24 years, who used methamphetamine in the last 90 days were more likely to have multiple partners $(85.7 \%)$ and to not use a condom every time $(66.7 \%)$. This was compared with individuals reporting no hard drug use $163.1 \%$ and $45.7 \%$, respectively).

17. Solomon TM, Halkitis PN, Moeller RW, Pappas MK. Levels of methamphetamine use and addiction among gay, bisexual, and other men who have sex with men. Addict Res Theory. 2012;20(1):21-9. The study found high levels of methamphetamine use among racial and ethnic minority MSM. Dependence on methamphetamine was highly associated with dependence on numerous other illicit drugs including cocaine, indicating that poly-drug use is common among MSM who use methamphetamine.

18. Young SD, Shoptaw S. Stimulant use among African American and Latino MSM social networking users. J Addict Dis. 2013;32(1):39 45.

19. Clark T, Marquez C, Hare C, John M, Klausner J. Methamphetamine use, transmission risk behavior and internet use among HIV-infected patients in medical care, San Francisco, 2008. AIDS Behav. 2012;16(2):396-403. The study reported an increased rate of unprotected sex among HIV-positive methamphetamine users. Among those who have used methamphetamine in the past 12 months, $27 \%$ of MSM reported unprotected sex sometimes, often or always compared with $10 \%$ who did not use the drug.

20. Nakamura N, Mausbach BT, Ulibarri MD, Semple SJ, Patterson TL. Methamphetamine use, attitudes about condoms, and sexual risk behavior among HIV-positive men who have sex with men. Arch Sex Behav. 2011;40(2):267-72. The study found that using methamphetamine 'to party' was one of four factors associated with use of the drug. This factor was associated with more aggressive unprotected sexual behaviors, including unprotected sex. Other reasons for the initiation of methamphetamine use was for selfesteem, to cope, and for energy.

21. Jerome RC, Halkitis PN, Coley MA. Methamphetamine use patterns among urban Black men who have sex with men. Cult Health Sex. 2009;11(4):399-413. The goal of this exploratory study was to investigate treatment strategies for Black MSM (BMSM) who may be used to inform methamphetamine treatment programs. Results indicated four treatment areas: (1) outreach/recruitment strategies; (2) therapist qualities; (3) group characteristics; and (4) intervention elements themselves. Findings underscore the importance of adapting evidence-based methamphetamine treatment strategies to include culturally relevant.

22. Vosburgh H, Mansergh G, Sullivan P, Purcell D. A review of the literature on event-level substance use and sexual risk behavior among men who have sex with men. AIDS Behav. 2012;16(6):1394-410. This study notes that methamphetamine use and binge drinking are consistently associated with sexual risk behavior among MSM. After controlling for other variables, methamphetamine use before sex 
continued to have a strong association with receptive anal intercourse without a condom.

23. Ghanem A, Little S, Drumright L, Liu L, Morris S, Garfein R. Highrisk behaviors associated with injection drug use among recently HIV-infected men who have sex with men in San Diego, CA. AIDS Behav. 2011;15(7):1561-9. This study found a high prevalence of methamphetamine use during sex among recently HIV positive individuals. The use of methamphetamine during sex was associated with multiple sex partners and unprotected anal intercourse among MSM.

24. Gorbach PM, Weiss RE, Jeffries R, Javanbakht M, Drumright LN, Daar ES, et al. Behaviors of recently HIV-infected men who have sex with men in the year postdiagnosis: effects of drug use and partner types. J Acquir Immune Defic Syndr. 2011;56(2):176-82.

25. Shoptaw S, Reback CJ, Peck JA, Yang X, Rotheram-Fuller E, Larkins S, et al. Behavioral treatment approaches for methamphetamine dependence and HIV-related sexual risk behaviors among urban gay and bisexual men. Drug Alcohol Depend. 2005;78(2): 125-34.

26. Reback CJ, Larkins S, Shoptaw S. Changes in the meaning of sexual risk behaviors among gay and bisexual male methamphetamine abusers before and after drug treatment. AIDS Behav. 2004;8(1):87-98.

27. Shoptaw S, Reback CJ, Larkins S, Wang P, Rotheram-Fuller E, Dang J, et al. Outcomes using two tailored behavioral treatments for substance abuse in urban gay and bisexual men. J Subst Abuse Treat. 2008;35(3):285-93.

28. Reback CJ, Shoptaw S. Development of an evidence-based, gayspecific cognitive behavioral therapy intervention for methamphetamine-abusing gay and bisexual men. Addict Behav. 2014;39(8):1286-91.

29. Reback CJ, Veniegas R, Shoptaw S. Getting off: development of a model program for gay and bisexual male methamphetamine users. J Homosex. 2014;61(4):540-53.

30. Garfein RS, Metzner M, Cuevas J, Bousman CA, Patterson T. Formative assessment of ARM-U: a modular intervention for decreasing risk behaviors among HIV-positive and HIV-negative methamphetamine-using MSM. Open AIDS J. 2010;4:105-15. This investigation was a formative research study used to evaluate the content and to assess feasibility and acceptability of this individual-level six-session HIV risk-reduction intervention for methamphetamine-using MSM. The intervention consists of six sessions. The objective of the formative research presented here was to evaluate the feasibility and acceptability of the ARMU intervention among MUMSM and identify ways to improve the intervention prior to conducting a pilot test of the intervention.

31. Zule WA, Poulton WE, Coomes CM, Mansergh G, Charania M, Wechsberg WM, et al. Results of a pilot study to reduce methamphetamine use and sexual risk behaviors among methamphetamineusing men who have sex with men (MSM) not currently in treatment. J Psychoactive Drugs. 2012;44(5):351-8. The purpose of this study was to develop and pilot test a single-session MI intervention for reducing HIV risk among an out-of-treatment sample of MSM who use methamphetamine. The MI was designed to increase selfefficacy of participants to avoid high-risk behaviors. Results suggest that a single-session MI intervention may be useful for reducing methamphetamine use and sexual risk among MSM who use methamphetamine, especially in settings where multisession interventions are not feasible.

32. Jerome RC, Halkitis PN. An exploratory investigation of treatment strategies for Black, gay, bisexual, and heterosexual men-whohave-sex-with-men who use methamphetamine. J LGBT Issues Couns. 2014;8(1):2-24

33. Wu E, El-Bassel N, McVinney LD, Hess L, Remien RH, Charania $\mathrm{M}$, et al. Feasibility and promise of a couple-based HIV/STI preventive intervention for methamphetamine-using, Black men who have sex with men. AIDS Behav. 2011;15(8):1745-54.

34. El-Bassel N, Witte SS, Gilbert L, Wu E, Chang M, Hill J, et al. The efficacy of a relationship-based HIV/STD prevention program for heterosexual couples. Am J Public Health. 2003;93(6):963-9.

35. Reback CJ, Peck JA, Dierst-Davies R, Nuno M, Kamien JB, Amass L. Contingency management among homeless, out-of-treatment men who have sex with men. J Subst Abuse Treat. 2010;39(3): 255-63. CM was implemented in a community HIV prevention setting and targeted reduced substance use and increased healthpromoting behaviors over a 24-week intervention period, achieving greater reductions in stimulant and alcohol use, in particular, methamphetamine use. Participants in the CM condition earned points for completing the targeted health-promoting behaviors and for drug/alcohol abstinence. Reductions in substance use were maintained to 9- and 12-month follow-up evaluations. Findings indicate the utility of $C M$ for this high-risk population and the feasibility of implementing the intervention in a community-based HIV prevention program.

36. Reback CJ, Ling D, Shoptaw S, Rohde J. Developing a text messaging risk reduction intervention for methamphetamine-using MSM: research note. Open AIDS J. 2010;4:116-22. The aim of this study was to conduct formative work to develop a text messaging intervention to reduce methamphetamine use and high-risk sexual behaviors among out-of-treatment MSM, and the development of a 2-week, text-messaging intervention that delivered realtime electronic correspondence based on the behavioral change theories of Social Support Theory, Health Belief Model, and Social Cognitive Theory.

37. Das M, Santos D, Matheson T, Santos G, Chu P, Vittinghoff E, et al. Feasibility and acceptability of a phase II randomized pharmacologic intervention for methamphetamine dependence in high-risk men who have sex with men. AIDS. 2010;24(7):991.

38. Braine N, Acker CJ, van Sluytman L, Friedman S, Jarlais DCD. Drug use, community action, and public health: gay men and crystal meth in NYC. Subst Use Misuse. 2011;46(4):368-80.

39. Halkitis PN, Wolitski RJ, Millett GA. A holistic approach to addressing HIV infection disparities in gay, bisexual, and other men who have sex with men. Am Psychol. 2013;68(4):261. This theoretical paper proposes a model for HIV prevention. It is suggested that HIV must be understood as one of a constellation of health problems facing gay and bisexual men-a syndemic. HIV prevention approaches must consider the impacts of biological, behavioral, social and psychosocial factors in addressing HIV and these other overlapping health conditions including substance use, violence, and other sexually transmitted infections.

40. Halkitis PN. Discrimination and homophobia fuel the HIV epidemic in gay and bisexual men. Director. 2012;202:6176.

41. Jerome RC, Halkitis PN. Stigmatization, stress, and the search for belonging in black men who have sex with men who use methamphetamine. J Black Psychol. 2009;35(3):343-65. This study examined factors related to methamphetamine use among Black MSM. Findings indicate that multiple forms of stress related to race, the possibility of contracting HIV, and internalized homophobia explain the use of the drug by these men.

42. Meyer IH. Prejudice, social stress, and mental health in lesbian, gay, and bisexual populations: conceptual issues and research evidence. Psychol Bull. 2003;129(5):674.

43. Semple S, Strathdee S, Zians J, Patterson T. Factors associated with sex in the context of methamphetamine use in different sexual venues among HIV-positive men who have sex with men. BMC Public Health. 2010;10(1):178-84.

44. VanDevanter N, Duncan A, Burrell-Piggott T, Bleakley A, Birnbaum $\mathrm{J}$, Siegel K, et al. The influence of substance use, social sexual environment, psychosocial factors, and partner characteristics on high-risk sexual behavior among young Black and Latino men who 
have sex with men living with HIV: a qualitative study. AIDS Patient Care STDS. 2011;25(2):113-21.

45. Marshall B, Wood E, Shoveller J, Patterson T, Montaner J, Kerr T. Pathways to HIV risk and vulnerability among lesbian, gay, bisexual, and transgendered methamphetamine users: a multi-cohort gender-based analysis. BMC Public Health. 2011;11(1):20-30. This investigation noted that sexual minority men and women are more likely than their heterosexual counterparts to use methamphetamine. Use of the drug was associated with injection drug use. Among males, use was also associated with unprotected sexual behaviors and depressive symptoms; among females, use of the drugs was associated with unprotected sex with sex trade clients.

46. Halkitis PN, Kupprat SA, Hampton MB, Perez-Figueroa R, Kingdon M, Eddy JA, et al. Evidence for a syndemic in aging
HIV-positive gay, bisexual, and other MSM: implications for a holistic approach to prevention and health care. Ann Anthropol Pract. 2012;36(2):365-86.

47. Siconolfi DE, Halkitis PN, Moeller RW. Homo economicus: young gay and bisexual men and the new public health. Crit Public Health. Epub 8 Apr 2014.

48. Halkitis PN. Reframing HIV, prevention for gay men in the United States. Am Psychol. 2010;65(8):752.

49. Institute of Medicine (US) Committee on Lesbian, Gay, Bisexual, and Transgender Health Issues and Research Gaps and Opportunities. The health of lesbian, gay, bisexual, and transgender people: building a foundation for better understanding. Institute of Medicine of the National Academy of Sciences; 2011:1-4 